\title{
Correction to: The early therapeutic response at 2 weeks is a crucial predictor of proton pump inhibitor-refractory gastroesophageal reflux disease
}

\author{
Maiko Ogawa' ${ }^{1}$ - Seiji Arihiro ${ }^{1} \cdot$ Nobuyuki Matsuhashi $^{2} \cdot$ Takashi Joh $^{3} \cdot$ Kazuhide Higuchi $^{4} \cdot$ Katsuhiko Iwakiri $^{5}$. \\ Takeshi Kamiya $^{6} \cdot$ Noriaki Manabe $^{7} \cdot$ Kimio Isshi $^{8} \cdot$ Tatsuya Nakada $^{1} \cdot$ Atsushi Hokari $^{1} \cdot$ Masayuki Saruta ${ }^{9}$. \\ Atsushi Oshio $^{10} \cdot$ Ken Haruma $^{11} \cdot$ Koji Nakada ${ }^{12}$
}

Published online: 8 April 2021

(c) The Japan Esophageal Society 2021

\section{Correction to: Esophagus (2021) 18:398-406 https://doi.org/10.1007/s10388-020-00792-z}

In the original publication of the article, Fig. 1a was published incorrectly. The number of Non-responder at 2 weeks was published as 11 . The correct value is 114 .

The correct Fig. 1 is provided below.

Publisher's Note Springer Nature remains neutral with regard to jurisdictional claims in published maps and institutional affiliations.

The original article can be found online at https://doi.org/10.1007/ s10388-020-00792-z.

\section{Koji Nakada}

nakada@jikei.ac.jp

1 Division of Gastroenterology and Hepatology, Department of Internal Medicine, Katsushika Medical Center, The Jikei University School of Medicine, Tokyo, Japan

2 Department of Gastroenterology, NTT Medical Center Tokyo, Tokyo, Japan

3 Gamagori City Hospital, Aichi, Japan

4 Second Department of Internal Medicine, Osaka Medical College, Osaka, Japan

5 Department of Gastroenterology, Nippon Medical School, Graduate School of Medicine, Tokyo, Japan

6 Department of Medical Innovation, Nagoya City University Graduate School Medical Sciences, Nagoya, Japan
7 Division of Endoscopy and Ultrasonography, Department of Laboratory Medicine, Kawasaki Medical School General Medical Center, Okayama, Japan

8 Isshi Gastro-Intestinal Clinic, Tokyo, Japan

9 Division of Gastroenterology and Hepatology, Department of Internal Medicine, The Jikei University School of Medicine, Tokyo, Japan

10 Faculty of Letters, Arts and Sciences, Waseda University, Tokyo, Japan

11 Department of General Internal Medicine 2, Kawasaki Medical School Kawasaki Hospital, Okayama, Japan

12 Department of Laboratory Medicine, The Jikei University School of Medicine, 3-25-8, Nishi-shimbashi, Minato-ku, Tokyo 105-8461, Japan 
Fig. 1 Responder rates at 2 and 4 weeks of PPI treatment. a Responder definition by GERD-SS residual symptom rate $\leq 50 \%$; b responder definition by patient's impression as "improved" or better (Q11); c responder definition by numeric rating scale $\leq 5(\mathrm{Q} 12)$. $P P I$ proton pump inhibitor; GERD-SS gastroesophageal reflux diseasesubscale; $S x$ symptom (a)
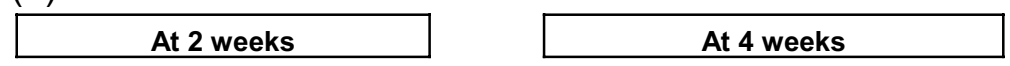

Responder at 2 weeks $n=181(61 \%)$

(Residual Sx rate, $20 \% \pm 20 \%$ )

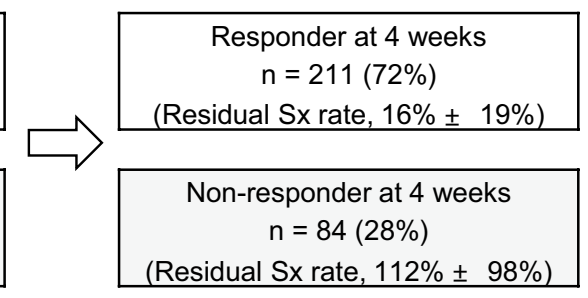

McNemar test $P=0.0006$

Non-responder at 2 weeks $\mathrm{n}=114(39 \%)$

(Residual Sx rate, $99 \% \pm 53 \%$ )

(b)

At 2 weeks

Responder at 2 weeks $\mathrm{n}=198(66 \%)$

(Residual Sx rate, $37 \% \pm 51 \%$ )

Non-responder at 2 weeks $\mathrm{n}=103(34 \%)$

(Residual Sx rate, $79 \% \pm 44 \%$ )

(c)

At 2 weeks

Responder at 2 weeks $n=230(76 \%)$

(Residual Sx rate, $39 \% \pm 50 \%$ )

Non-responder at 2 weeks $\mathrm{n}=71(24 \%)$

(Residual Sx rate, $87 \% \pm 44 \%$ )

\section{At 4 weeks}

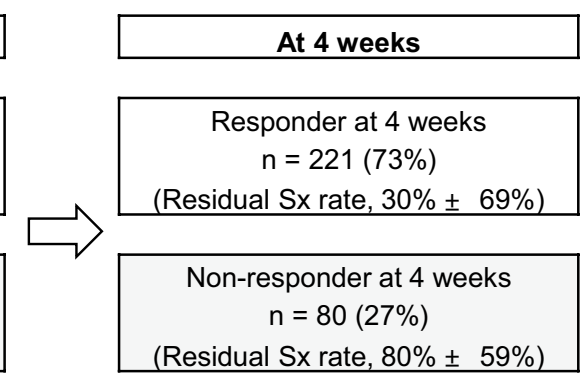

McNemar test $P=0.0038$
McNemar test $P<0.0001$ 